\title{
Temperature Mapping of a Re-entry Vehicle Flap in High Enthalpy Flow Test
}

\author{
by T. Rösgen*, C. Pereira** S. Airaghi* and A. Vuilleumier** \\ *Institute of Fluid Dynamics, ETH Zurich, 8092 Zurich, Switzerland, roesgen@ifd.mavt.ethz.ch \\ ${ }^{* *}$ RUAG Space, Schaffhauserstr. 580, 8052 Zurich, Switzerland, carlos.pereira@ruag.com
}

\begin{abstract}
The paper describes the results of a joint test campaign in the "Scirocco" high enthalpy flow facility. The tests were aimed at validating a system to measure the rear surface temperatures on the EXPERT re-entry vehicle's flaps using near infrared thermography and a set of supporting reference thermocouples. The optical system consists of a sapphire lens assembly placed behind the flap, a fiber optic image guide and an uncooled near-infrared camera. In order to provide data redundancy and an independent means of verification, an external infrared camera was used to record the external surface temperatures as well.
\end{abstract}

\section{Introduction}

The detailed understanding of aero-thermodynamical phenomena is essential in the development of spacecraft designed for atmospheric re-entry. A promising approach to achieve this objective is the combination of flight experiments, numerical simulations and validation in ground test facilities. This is exemplified in the European eXPErimental Reentry Testbed ("EXPERT") project of the European Space Agency (ESA), where a sub-orbital flight and atmospheric re-entry of a fully instrumented capsule (see figure 1) is to be performed to collect relevant thermo-physical data [1]. Numerical models of the spacecraft performance can then be checked for the validity of the inherent model assumptions.
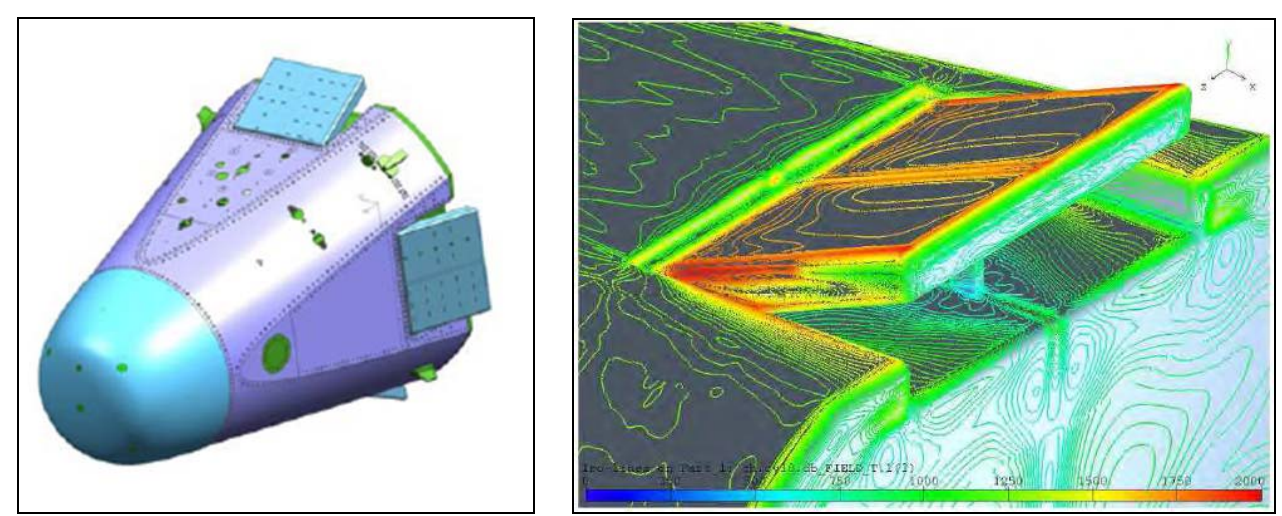

Fig. 1. The EXPERT Vehicle: CAD model (left, [2]) and simulated flow field (right, [3])

As part of the preparation for the actual flight, full-scale ground tests were performed in the high enthalpy plasma wind tunnel "Scirocco", jointly built by ESA and the Italian National Aerospace Research Center (CIRA) $[4,5]$. The flow field and thermal behavior on one of EXPERT's static flaps were studied during exposure to heat loads representative of the vehicle's re-entry flight profile.

The sensors belonged to three different payload experiments on the EXPERT vehicle. Payload experiment \#7 is operated by CIRA and mainly concerned with the flow characterization upstream of the flap as recorded by heat flux sensors. Payload experiment \#6, designed and operated by the German Aerospace Establishment DLR, consists of several thermocouples and pressure sensors installed on the flap surface. Duplicating the flight configuration, the test article was furthermore instrumented with a ruggedized infrared camera recording the rear surface thermal radiation of the flap. This camera forms the centerpiece of EXPERT's scientific payload experiment \#8 developed and operated by RUAG Space for the European Space Agency.

The rear surface camera was augmented by a second IR camera (provided by CIRA) which monitored the outside surface of the flap. During the ground tests, the two infrared cameras measuring inner and outer surface temperatures allow to obtain a direct estimate of the heat fluxes through the flap.

In contrast, the payload 8 camera will be used alone during the flight to record the rear flap surface temperatures, because external viewing is not possible. Using a suitable thermal model of the flap, it will then be attempted to infer the flap outside surface temperatures. The Scirocco test objectives were selected to validate this "inverse mapping" approach and to support the numerical simulation of the mission. 


\section{Experimental campaign}

A full-scale replica of one of the flaps of the EXPERT vehicle was mounted on a cooled sample holder inside the Scirocco plasma wind tunnel (see figure 2).

This facility is capable of generating a continuous plasma flow heated by a $70 \mathrm{MW}$ electric arc. Air temperatures up to $10,000^{\circ} \mathrm{C}$ can be reached. These specifications, together with the dimensions of the vacuum test chamber and the plasma jet ( $2 \mathrm{~m}$ diameter) make Scirocco the largest and most advanced plasma tunnel in the world.

Reflecting the different payload experiments, the Scirocco measurement campaign was conducted as a joint test activity between payload 6 and 8 and coordinated by ESA. Using the expertise and instrumentation of all the payload providers and that of the facility operator a complete and redundant measurement set could be acquired for all test runs.

CIRA operated the facility and recorded the necessary housekeeping data. In addition, it operated the external infrared camera and processed its image data. RUAG Space built the test article and equipped it with the rear surface infrared camera and data handling system. (In fact, the hardware used was the "qualification model" for the EXPERT mission flight hardware.) The German Aerospace Establishment (DLR) was responsible for installing the reference thermocouples on the flap and for the post-processing of their data.

\subsection{Test article}

The EXPERT flap was installed on a water-cooled sample holder inside the Scirocco test section as shown in figure 2 (left). The holder was large enough to house also the on-board data handling unit (DHU), facilitating the use of the EXPERT flight hardware design without modifications.

The flap was made of Keraman® $\mathrm{C} / \mathrm{SiC}$ at MT Aerospace and had a size of $323 \mathrm{~mm} \times 396 \mathrm{~mm}$.
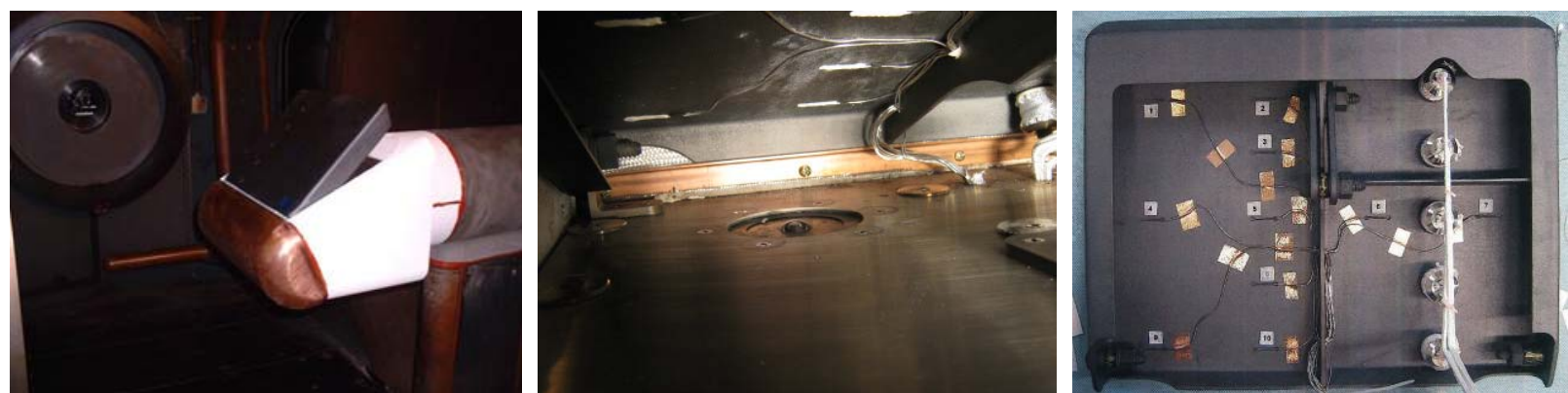

Fig. 2. EXPERT flap test article [4] (left: outside view; center: view of rear cavity; right: rear view of flap surface)

In the center part of the figure, a view into the flap rear cavity is shown. The reference thermocouples attached to the flap's rear surface and the necessary wiring harness are visible. On the bottom surface of the cavity (corresponding to the EXPERT vehicle body surface) the front end lens of the in-flight infrared camera system can be recognized.

\subsection{Rear surface infrared camera and optics}

In order to measure the rear surface temperatures, the payload 8 design employs a hybrid infrared imaging system. A near-infrared camera records the radiation from the surface, while a set of thermocouples registers the temperatures independently at several reference points. Matching the infrared signatures with those reference temperatures, a full field calibration of the infrared camera is achieved without knowledge of the flap's surface emissivity.
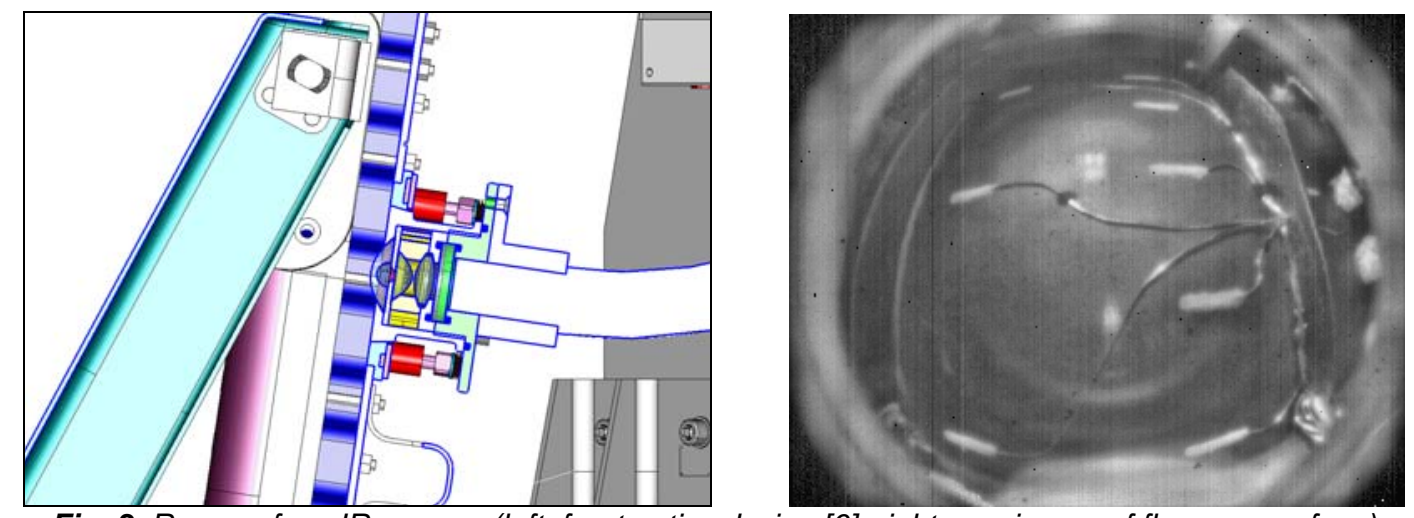

Fig. 3. Rear surface IR camera (left: front optics design [6], right: raw image of flap rear surface) 
The imaging system consists of a wide angle front lens optics assembly based on three custom sapphire lenses in a heat-resistant assembly. An intermediate image is transmitted via a $50 \mathrm{~cm}$ fiber optic image guide to a relay lens assembly which includes an exchangeable optical narrow bandpass filter (center wavelength $1670 \mathrm{~nm}$ ). The relay lens system maps the image onto the sensor surface of the infrared camera (SWIR un-cooled InGaAs array with $320 \times 256$ pixels resolution).

The front optics is designed to capture the complete inner flap surface area in a single image. This necessitates the recording of a significantly distorted scene, as exemplified in figure 3 . In the image (created here with artificial lighting for better visibility) the reference thermocouples, flap center divider and the DLR pressure sensors can clearly be identified.

\subsection{Data acquisition system}

The camera images are transferred at a frame rate of $25 \mathrm{~Hz}$ to a dedicated data handling unit (DHU) using a USB 2.0 data link. The images are stored in full resolution and bit precision (14 bit) in the DHU's non-volatile memory. In addition to this primary function, the DHU performs several other tasks in real-time. Different house-keeping temperatures (e.g. those of the hot optical frontend and the IR camera) are logged, and the IR images are analyzed for signs of over- or under-exposure. If such a condition is detected, the camera's exposure time and gain are adjusted automatically to provide an optimal image contrast.

\subsection{Test Sequences}

During the test campaign in the plasma wind tunnel, four runs were performed, corresponding to different reentry trajectories of the EXPERT vehicle. The parameters of the runs are listed in Table 1.

Table 1. Parameters of Scirocco test campaign

\begin{tabular}{|c|c|c|}
\hline Run \# & Power [MWi & Duration [sec] \\
\hline 1 & 38 & 110 \\
\hline 2 & 38 & 110 \\
\hline 3 & 28 & 140 \\
\hline 4 & 28 & 140 \\
\hline
\end{tabular}

For each run, the following data were recorded and merged for the final analysis.

Table 2. Sensors used during test campaign

\begin{tabular}{|l|l|l|}
\hline \multicolumn{1}{|c|}{ Sensor Type } & \multicolumn{1}{c|}{ Provided / recorded by } & \multicolumn{1}{c|}{ Comment } \\
\hline Flap rear surface IR images & ESA EXPERT P/L 8 IR camera & Flight hardware \\
\hline Flap front surface IR images & CIRA external IR camera & \\
\hline Flap rear surface thermocouples & DLR & \\
\hline Front optics thermocouples & ESA EXPERT P/L 8 housekeeping & also recorded by CIRA \\
\hline
\end{tabular}

\section{Data Analysis}

The aim of the various data analysis steps is twofold. First, the rear surface infrared images are to be converted into rectified images of the rear surface temperature. This part of the analysis is representative of the data products to be generated from the in flight data as well.

In addition, a comparison and co-registration of these rear surface temperature data is performed with front surface data available from the external IR camera operated outside the plasma wind tunnel. By comparing temperatures at the same point on the flap's front and rear surface an estimate for the local heat flux as a function of space and time can be derived.

Four data processing groups can be distinguished according to Table 2. The data in each group have their own processing requirements and all of them need to be aligned in time, as no master time reference was available.

The EXPERT payload 8 infrared camera images were processed with the following steps, after the images were off-loaded from the onboard memory.

- Offset (fixed pattern noise) correction for each pixel based on calibration data,

- Gain (flat field) correction for each pixel based on calibration data,

- Scaling of the pixel values ("digital levels") to temperatures based on reference thermocouple readings,

- Image rectification and interpolation onto the flap reference coordinate system, and

- Extraction of temperature values at the positions of the external IR camera's sampling points and lines.

Figure 4 shows the result of this processing for a typical image frame. On the left, a raw image is shown as stored on the payload $8 \mathrm{DHU}$. The right image shows the same scene, now rectified and converted to temperature values, here coded in false colors to enhance the visual contrast. In order to preserve the original data quality, the image 
rectification, which consists in a radial distortion correction [7] and a perspective re-mapping [8] ("homography"), is performed together with the re-sampling onto the reference grid as a single interpolation step. No attempts were made to correct (e.q. replace or interpolate) "bad" pixels in the raw images.
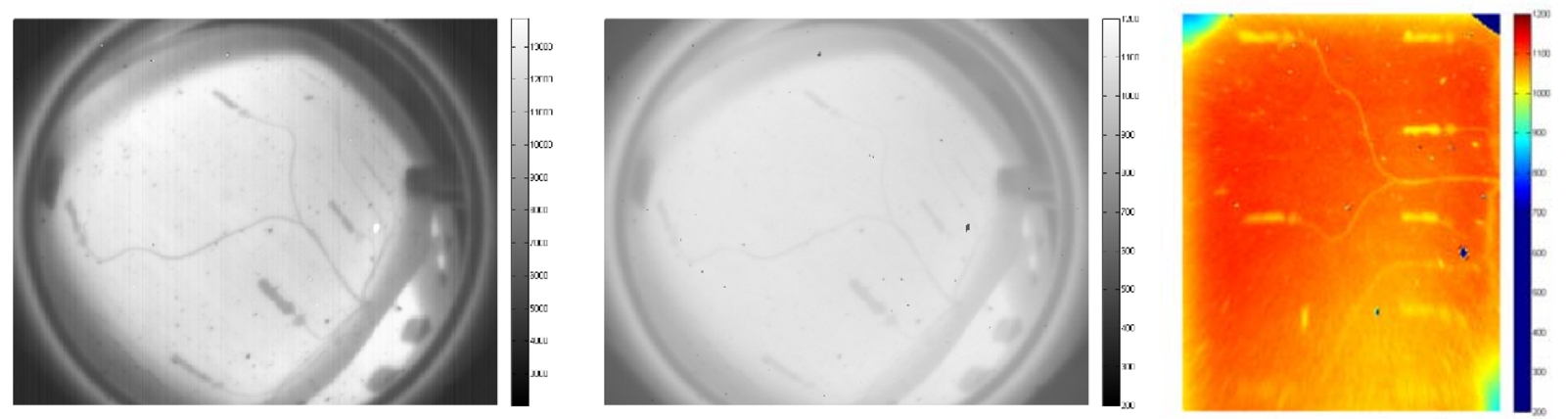

Fig. 4. Processing of IR images: left - raw; center - temperatures; right - rectified (false color coding)

The CIRA external camera's infrared images were delivered with the conversion to temperatures already performed. In particular, the temperature values at 16 specific sampling points and on 7 sampling lines were available (see figure 5).
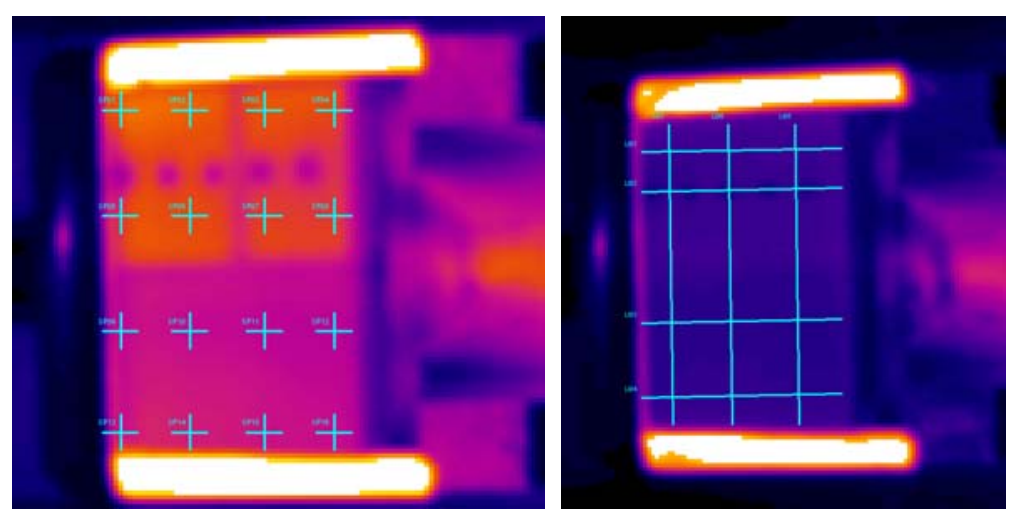

Fig. 5. External IR camera false color images [4] with marked sampling points (left) and sampling lines (right)

In order to compare those specific surface temperatures with the EXPERT payload 8 data, a geometric mapping of the sampling positions is performed. This mapping provides the coordinates of the CIRA data points in a global flap coordinate system (figure 6). The processing involves the following two steps.

- Extraction of the positions (in pixel coordinates) of the sampling points and lines in the external IR camera images, and

- Computation of the coordinate transforms to map the sampling points onto the flap coordinate system, based on identified flap features (pressure port locations, rear edge location, etc.).

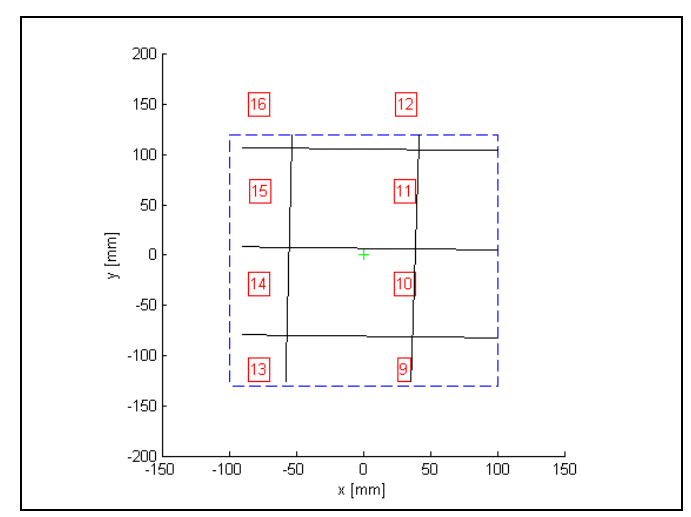

Fig. 6. Mapping of external camera sampling points (red) and lines (black) onto rear camera field-of-view (blue) 
Note that in the chosen coordinate system, the positive x-axis points towards the flap centerline, and the positive $y$-axis point towards the rear end of the flap.

The flap thermocouples provide the temperatures to calibrate the rear surface IR camera. This involves the computation of a scale / conversion factor from the camera's digital levels to true temperatures. Seven thermocouples are in the camera's field of view, and the scale factor is computed by performing a non-linear least squares fit between the measured temperature values and the associated pixel readings based on Planck's radiation formula.

Since the thermocouples have an infrared signature different from that of the surrounding surface (see figure 4), the temperatures are matched to the median pixel value on a sampling circle surrounding the actual thermocouple measurement point.

Finally, the experiment times between the external recordings (CIRA camera, DLR thermocouples) and the internal time scale of the EXPERT payload $8 \mathrm{DHU}$ have to be synchronized. This is done in two different ways.

- Correlation of the front optics temperature as simultaneously recorded by DLR and the IR camera DHU is used to align the DLR and payload 8 times.

- $\quad$ Correlation of the temporal temperature gradients as evaluated by the CIRA and EXPERT infrared cameras at the sampling points is used to align the CIRA and payload 8 times.

\section{Results and Discussion}

The simultaneous profiles of the front and rear temperatures at the sampling points SP9-SP11 and SP13-SP15 (cf. figure 6) are shown in figure 7. The experiment (here: Run 2 according to table 1) starts with a quiescent phase while the test article is still kept outside the hot plasma jet. At time $t=0$ the insertion into the beam is completed, and the surface temperatures rise rapidly. At time $t \approx 110$ s the plasma jet is switched off, and the test article slowly cools down while being exposed to a cold, protective nitrogen atmosphere.

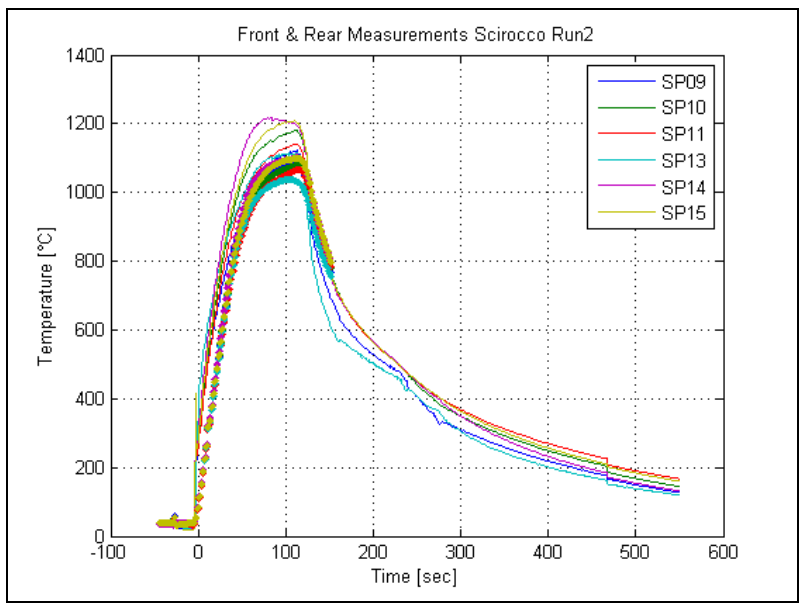

Fig. 7: Front (continuous) and rear (points) surface temperatures

Distinct spatial non-uniformities can be seen in the full rear surface temperature distributions at different times. Figure 8 shows that the flap initially heats fastest on the front outside edge. Conversely, the slowest heating can be observed at the back end of the flap towards the centerline.

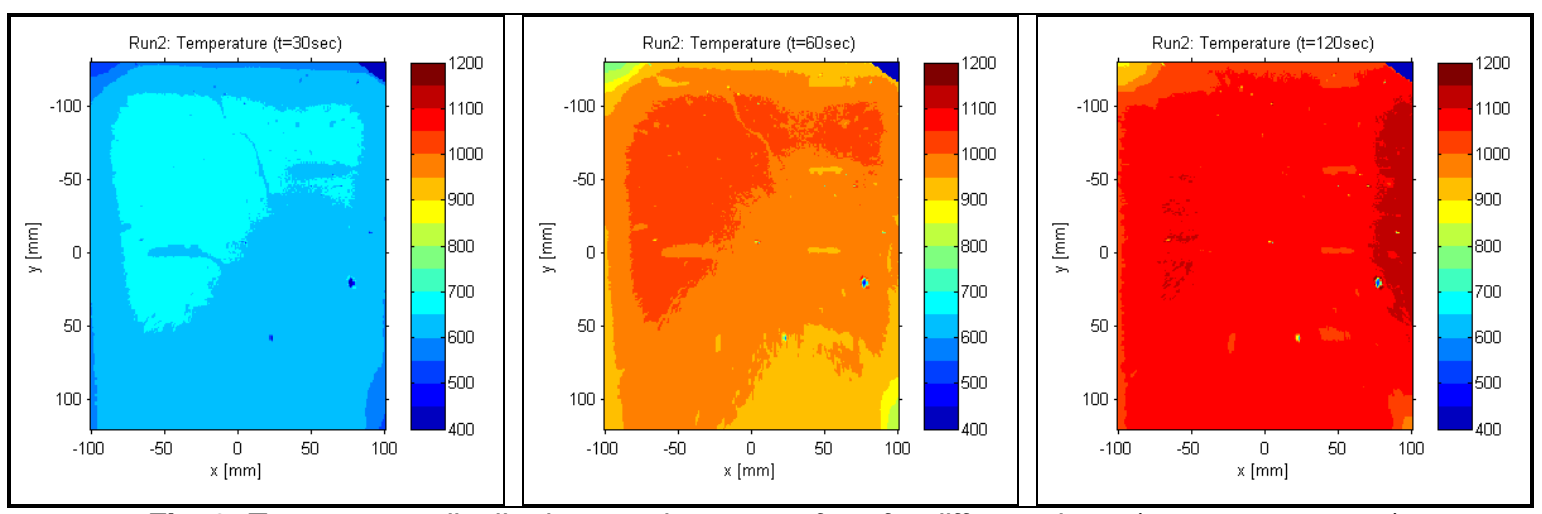

Fig. 8: Temperature distributions on the rear surface for different times ( $t=30,60,120$ sec.)

In order to further document significant features of the temperature evolution, the difference is computed between the front and rear surface temperatures at the selected measurement stations (figure 9). (Note that this 
difference is somewhat sensitive to the accuracy of the temporal alignment, especially in the early phase of rapid temperature increase.)

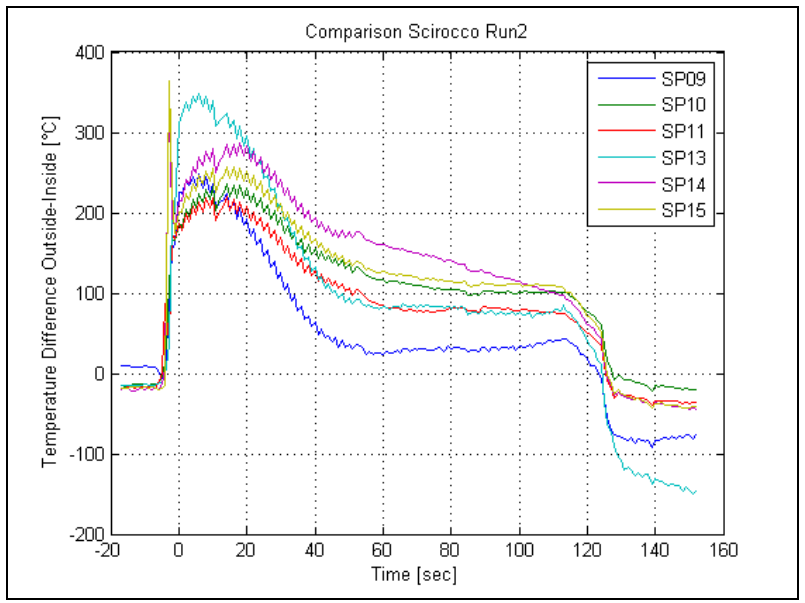

Fig. 9: Temperature differences (front - rear) at sampling points

The following observations can be made.

- First, one recognizes that there is a negative temperature difference at all measurement points during the cooldown phase of the experiment. This means that there is a heat flux towards the front surface, which is plausible because the flap outside surface is being cooled.

- During the heating and constant flow phase of the experiment, the sampling points show a positive temperature difference, implying a heat flux towards the flap rear surface and thus continued heating from the outside.

- Next, one may notice a somewhat different pattern for sampling points SP09 and SP13 compared to all others. These two sampling points are closest to the flap's front edge and may lie in a potentially different flow regime.

- $\quad$ Furthermore, if one compares the sampling points SP09, SP10 and SP11 with points SP13, SP14 and SP15 one observes that the points in the first group, which lie close to the flap's centerline, always indicate lower heat fluxes than those of their correspondence in the other group closer to the side of the flap.

- During the cooling phase, a cross-over can be noticed in the traces of SP09 and SP13, indicating that the heat fluxes in the flap's outside front corner tend to be larger than those on the front centerline, independent of their sign / direction.

- Finally, the temporal evolution of the temperature differences appears to level off during the heating phase. This means that the heat fluxes may have reached a steady state value while the actual temperatures (both on the flap's outside and rear surface) still keep increasing.

Changes in the temperature along the flap can also be recognized in the plots of the line scan profiles for lines LIO3 and LIO4 (figure 10, left) for an early time in the heating phase (t=30 sec.). The temperatures towards the flap front edge (negative y-values) are somewhat higher than those towards the rear edge (positive y). At the same time, the temperatures in the spanwise direction (lines LI05-LI07) remain roughly constant.
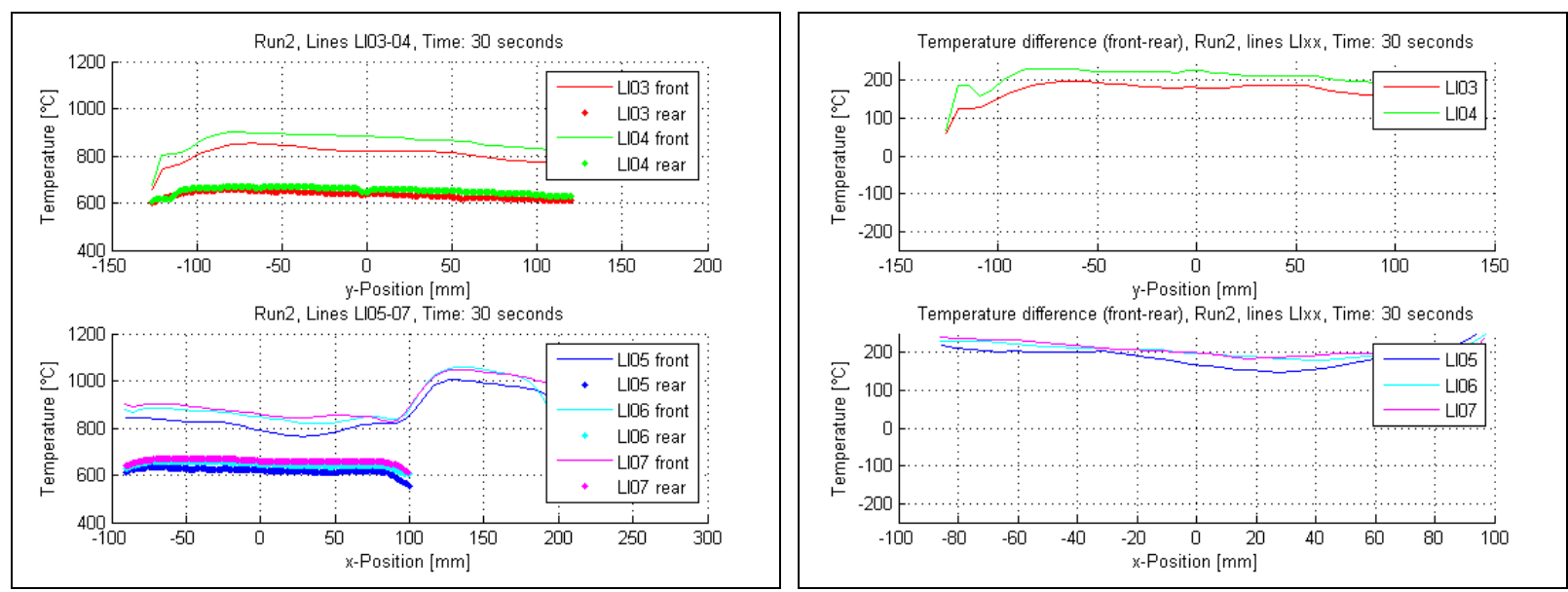

Fig. 10. Rear surface temperatures (left) and temperature differences (right) along sampling lines (t=30s) 
The heat fluxes (i.e. temperature differences, figures 10-12, right) remain constant in the streamwise direction and show a small decline towards the flap centerline. The same trend is recognizable at later times $(t=60 \mathrm{sec}$, figure 11) as well, although the temperature differences have decreased in magnitude.
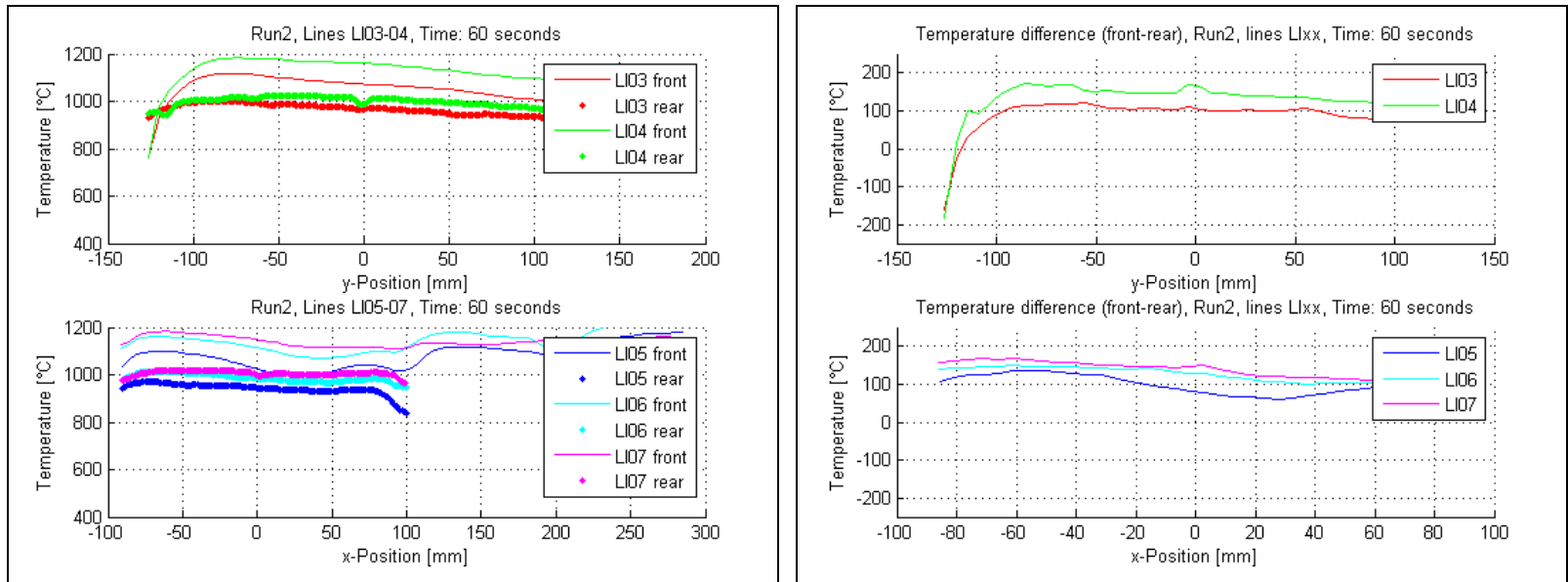

Fig. 11. Rear surface temperatures (left) and temperature differences (right) along sampling lines ( $t=60 \mathrm{~s})$

At $\mathrm{t}=120 \mathrm{sec}$. (figure 12) the temperature differences between the flap front and rear have become even smaller, indicating convergence towards a state with comparatively small heat fluxes.
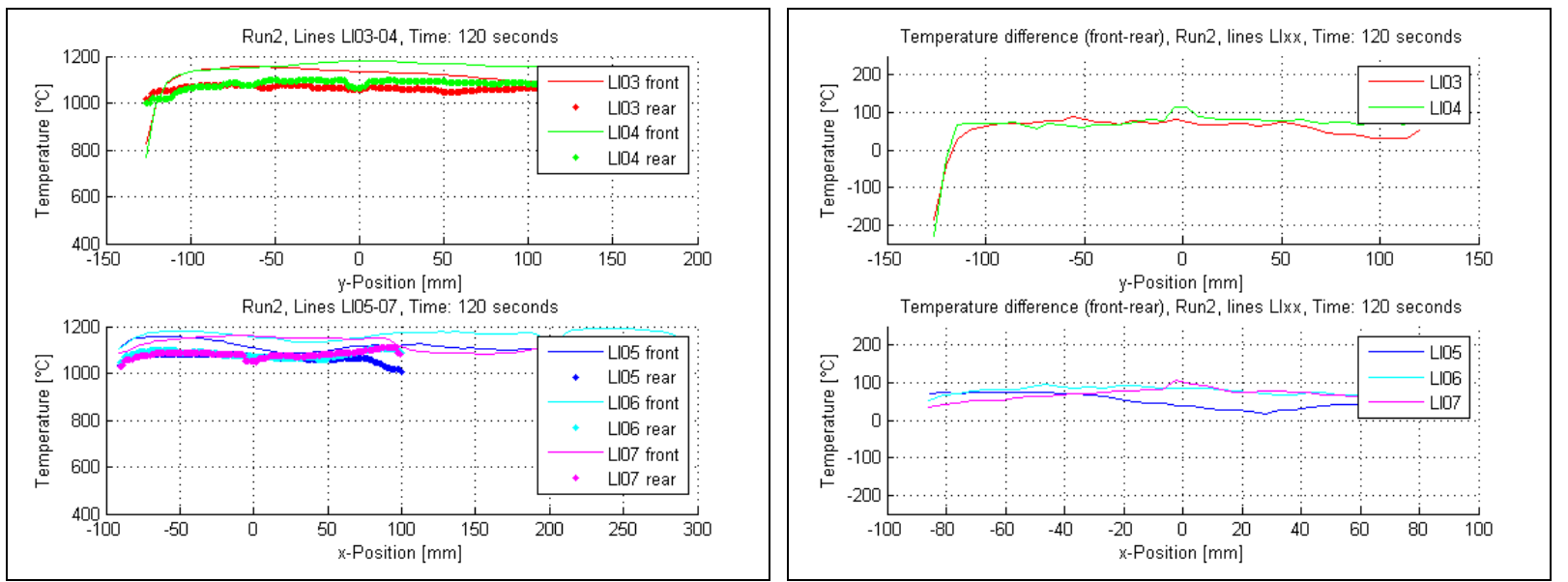

Fig. 12. Rear surface temperatures (left) and temperature differences (right) along sampling lines ( $t=120 \mathrm{~s})$

The temporal evolution of the rear surface temperatures can further be rendered as a space-time plot. Figure 13 presents the result for the streamwise line profiles and figure 14 provides those for the three spanwise sampling line sets.
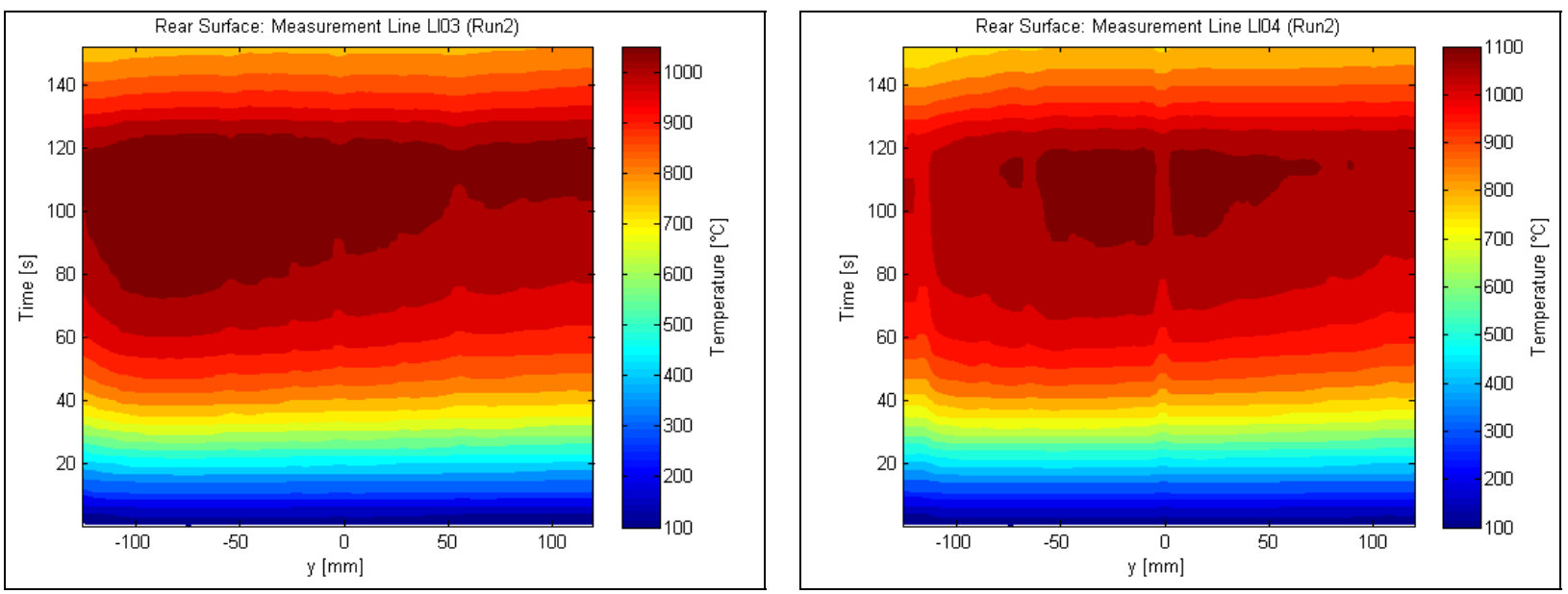

Fig. 13. Surface temperature evolution along streamwise sampling lines LIO3 and LIO4 
It becomes once again apparent that in general the largest gradients in any direction occur during the rapid heating phase, whereas a spatial equilibrium appears to be established later on. On the other hand, the heating up of the flap as a whole appears to continue right up to the time of beam shutdown.
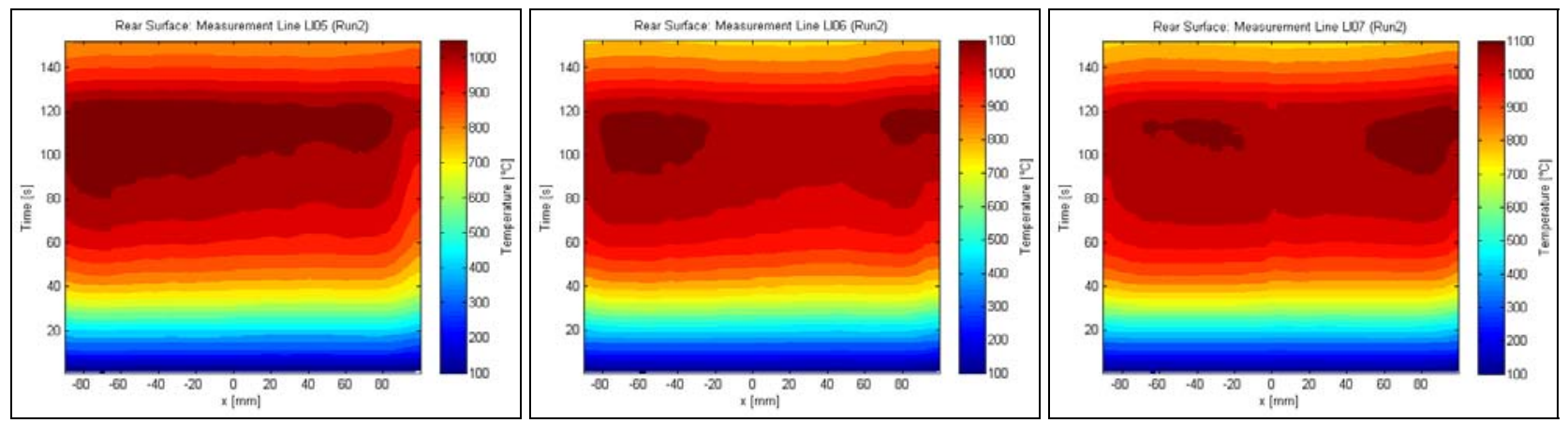

Fig. 14. Surface temperatures along spanwise sampling lines LIO5-LIOT

Similar results were also observed during the other three test runs. As a further example, figure 15 shows the evolution of the rear surface temperatures and the temperature differences for the third run which lasted approximately 30 seconds longer than the first and second one, but at a reduced jet thermal energy ( $28 \mathrm{MW}$ vs. $38 \mathrm{MW}$ ).
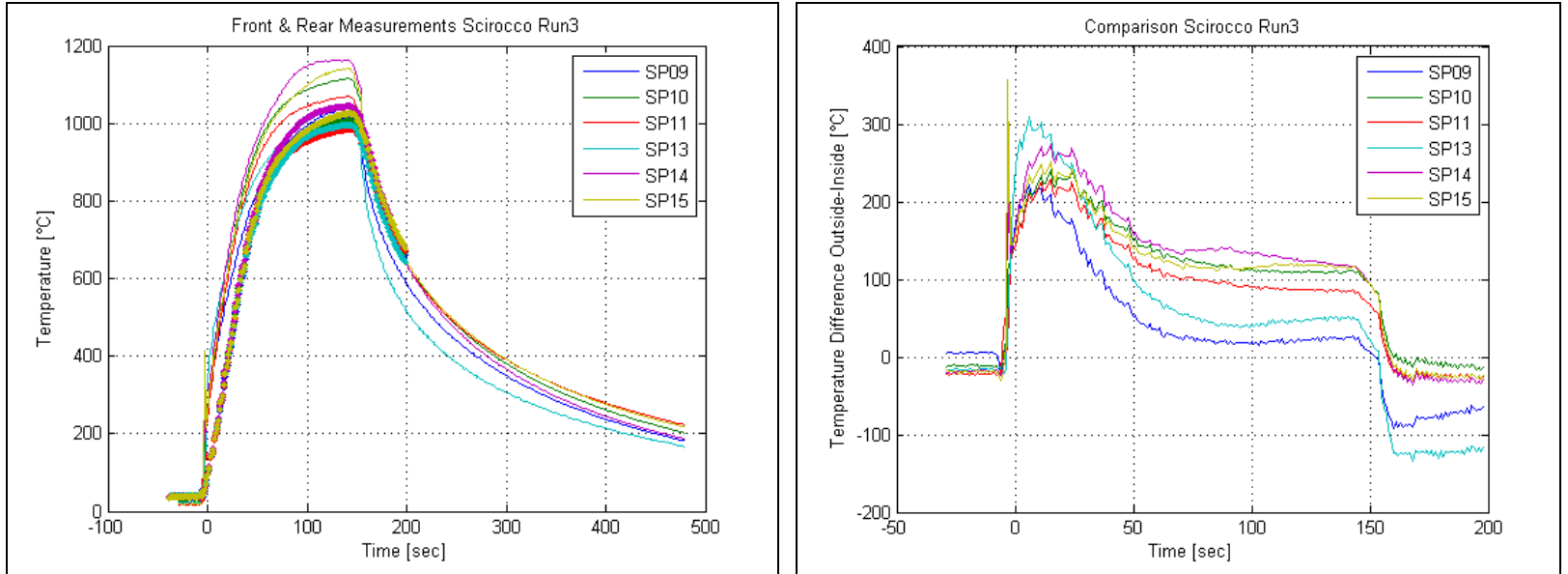

Fig. 15. Temperature and temperature difference evolution during long duration run

The overall thermal behavior is comparable to that of the second run, the only difference being the lower peak temperatures due to the reduce jet power. Again, there is no limit visible in those peak temperatures, while the temperature differences seem to have stabilized before the shutdown occurs.

\section{Outlook}

The test campaign successfully demonstrated the acquisition of surface temperatures on a re-entry vehicle flap based on infrared images. Using simultaneous recordings both on the front and rear surface, an overview of the spatial variation in the heat flux could be derived. This data will be used both to verify the results of a matching numerical simulation and to derive a model for inverse temperature mapping.

This latter technique will be used for the in-flight data analysis, where the outer surface temperatures will not be measured directly but have to be derived from the rear surface data alone based on a thermo-mechanical model of the flap and the outside flow field.

\section{Acknowledgements}

The authors wish to thank the Swiss Space Office and the European Space Agency for their support as well as the Institute of Aerodynamics of the German Aerospace Center in Cologne (DLR) and the Italian National Aerospace Research Center (CIRA) in Capua for the collaborative effort. 


\section{REFERENCES}

[1] Thoemel J., Muylaert J.M., Ratti F., Gavira J., "In-Flight Testing of Critical Technologies and Experimentation of Aerothermodynamic Phenomena". 16th AIAA/DLR/DGLR Int. Space Planes and Hypersonic Systems and Technologies Conf., AIAA 2009-7232, Bremen (Germany), Oct. 19-22, 2009

[2] http://www.esa.int/images/Expert_vehicle.jpg

[3] Pereira C., Hirtz B., Vuilleumier A., Roesgen T., Vos J., "Inverse Temperature Mapping of Re-Entry Vehicle Control Surfaces using Infrared Thermography". Proc. 6th European Symposium on Aerothermodynamics for Space Vehicles, ESA SP-659, Versailles (France), Nov. 3.6, 2008

[4] Trifoni E., Del Vecchio A., Di Clemente M., De Simone V., Martucci A., Purpura C., Savino L., Cipullo A., "Design of a Scientific Experiment on EXPERT Flap at CIRA SCIROCCO Plasma Wind Tunnel". Proceedings of the 7th European Symposium on Aerothermodynamics, ESA SP-692, Brugge (Belgium), May 9-12, 2011

[5] Gülhan A., Pereira C., Di Clemente M., Fertig M., Vos, J., "Aerothermodynamics of EXPERT Control Surfaces". Proc. 7th European Symposium on Aerothermodynamics, ESA SP-692, Brugge (Belgium), May 9-12, 2011

[6] Pereira C., Vuilleumier A., Handrick K., Roesgen T., Airaghi S., Vos, J., "Thermal Measurement Techniques for (Movable) Control Surfaces in Re-Entry Vehicles". Proc. 6th European Workshop on Thermal Protection Systems and Hot Structures, Stuttgart (Germany), Apr. 1-3, 2009

[7] Wang A., Quu T., Shao L., "A Simple Method of Radial Distortion Correction with Centre of Distortion Estimation". J. Math. Imaging Vis. 35, 165-172, 2009

[8] Hartley R.; Zisserman A., "Multiple View Geometry in Computer Vision". Cambridge University Press, 2003 